\title{
OPTIMAL SIGNAL CONDITIONING IN THE NIR MOISTURE METER
}

\author{
Mirko Filic, Venco Corluka, Zdravko Valter \\ Faculty of Electrical Engineering, University of Osijek, Croatia \\ Kneza Trpimira 2b, HR-31000 Osijek \\ E-mail: mirko.filic@etfos.hr
}

\begin{abstract}
The infrared radiation measurement system in the moisture meter based on the cooled PbS photoconductive detector is designed by the authors and analysed in this paper. It consisted of a preamplifier and a mechanical chopper. The basic noise sources and their influence on this measuring system are studied out. One solution for the maximisation signal to noise ratio based on the theoretical and laboratory practical consideration has also been made. Under the circumstances, the possible accuracy of the mentioned system is founded out by the measurement. The final aim is to develop an optimal optoelectronical measuring system for the infrared moisture meter for practical use but with some specific substances firstly. The overall results can be kept in the numerous variety applications later.
\end{abstract}

Key words: moisture meter, NIR, near infrared, noise, PbS detector

\section{INTRODUCTION}

The measurement of the moisture content of various industrial and non-industrial products, in component parts of agricultural products, for example, is of great importance for diagnostics and quality control. Just as the moisture can be measured more accurately in the drying processes, the desired product quality and the extra thermal energy saving can also be achieved in a more precise way. By applying the optoelectronical moisture meter in the automated manufacturing processes, the appropriate measuring promptness with a very high accuracy can be achieved [1]. The moisture measuring can be realized via reflected radiation at specific wavelengths in near infrared region (NIR) [3]. It has been established that NIR, although being inappropriate for the qualitative spectrometry, can at the same time be very acceptable for the quantitative analytics of some individual substance kinds including water [2]. The substance containing it has a great impact in that kind of moisture measuring and it makes the measuring to be more complex.

\section{MEASURING SYSTEM IN THE INFRARED MOISTURE METER}

The solution of a NIR moisture meter with one beam and two beams is possible [3]. Although the solution with two beams can eliminate some negative influence factors like the influence of sample surface, the particle size and the sample colour, moisture meter with one beam shown in Fig.1 is chosen due to its lower expenses. The characteristics of $\mathrm{PbS}$ photoconductive detectors such as high detection levels in wavelengths of between $1000 \mathrm{~nm}$ and

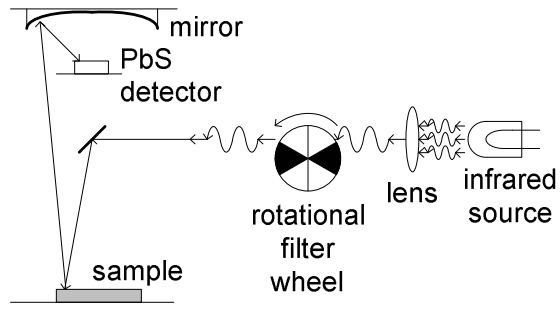

Fig. 1. Infrared moisture meter with one ray

$3200 \mathrm{~nm}$, good response speed and reasonable price, mean that they can be used in various applications for the moisture detection due to one distinctive absorption wavelengths of water at 1940nm [2]. The measurement system mechanically chops the radiation so the response time is limited by the chopping frequency. The bias voltage for the supply detector and the other active elements for the conditioning signal (Fig. 2) are obtained by the stabilized rectifier supply module from the AC power line. The small AC signals from the detector are

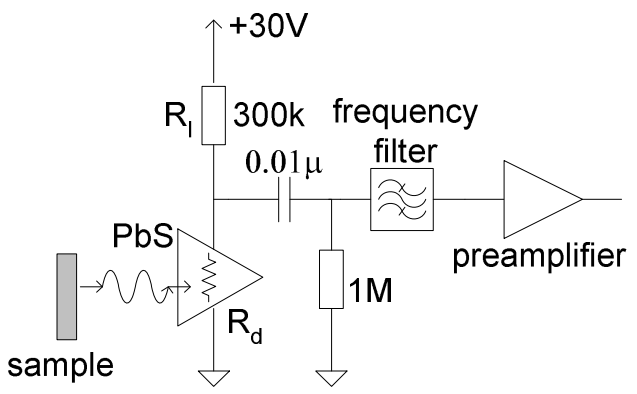

Fig. 2. Conditioning $\mathrm{PbS}$ detector signal 
conditioned by the RC coupled processing circuit which consisted of two different stages. The power line generated noise $(50 \mathrm{~Hz})$ is filtered by the first stage, the frequency filter. The useful signal is amplified by the second stage, the narrow bandwidth preamplifier.

\section{GENERAL NOISE MODEL}

Four various types of noise sources are perceived in proposed measuring system. It is the noise generated by the power supply module, background (photon) noise, detector noises and noise of signal processing circuit. All active elements such as the operational amplifiers of the preamplifier and frequency filter are supplied by rectifier module from $\mathrm{AC}$ power source. The infrared source, the drive of filter wheel and thermoelectrical cooler in the detector are supplied from the same power supply module, too. Consequently this module comprises six different rail voltage-current levels. Although the separate outputs from the power supply module are stabilized, the harmonic noises is either appeared from the AC power source or produced by the supply module. Odd multiples of the power line frequencies are usually expressed. These noises have the same level as is the voltage signal from the detector and therefore have been filtered by the frequency filter. Further noise filtering is executed by the choice of the mechanical chopper frequency. This is accomplished by means of the rotary speed of filter drive adjustment. The signals versus noise characteristics of the detector as well as the impact of the power line generated noise to the operating circuit require a choice of higher frequency. One common practice is applied, removing power line generated noise from the system so that the chopping is adjusted at an odd multiple of $1 / 2$ the line frequency of the power source.

The general noise model of the detector-processing circuit has been accepted and shown in Fig. 3. consists of the signal current source $I_{p h}$, the temperature dependable dark resistance $\mathrm{R}_{d}(\mathrm{~T})$ and the equivalent capacity $C_{d}$. The load resistance of the detector is shown by $\mathrm{R}_{1}$, as well as background noises and thermal noises of detector by the current generators $I_{b n}$ and $I_{t n}$ respectively. Typical $R_{d}(T)$ characteristic is shown in Fig.4. As active elements of the frequency filter and preamplifier operational amplifiers are used. These circuits' noises are presented by the voltage $V_{p c}$ source and by current $I_{p c}$ source. The input capacity and resistance of the processing circuit (the operational amplifier) are presented by the equivalent capacity $C_{\text {in }}$ and

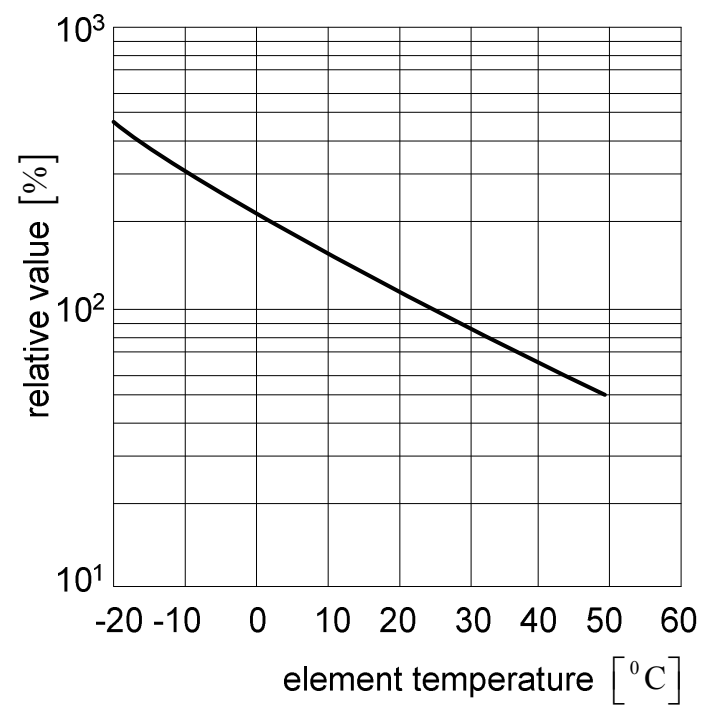

Fig. 4. Typical $R_{d}(T)$ characteristic

resistance $R_{\text {in }}$. The second specified noise type the background generated noise assumes the appearance as a radiance effect at an absolute temperature of over $0 \mathrm{~K}$. All objects with an absolute temperature of over $0 \mathrm{~K}$ emit infrared radiation. Infrared radiation energy is determined by the temperature and surface condition of an object. The background noise can not be estimated exactly. In this moisture meter, the

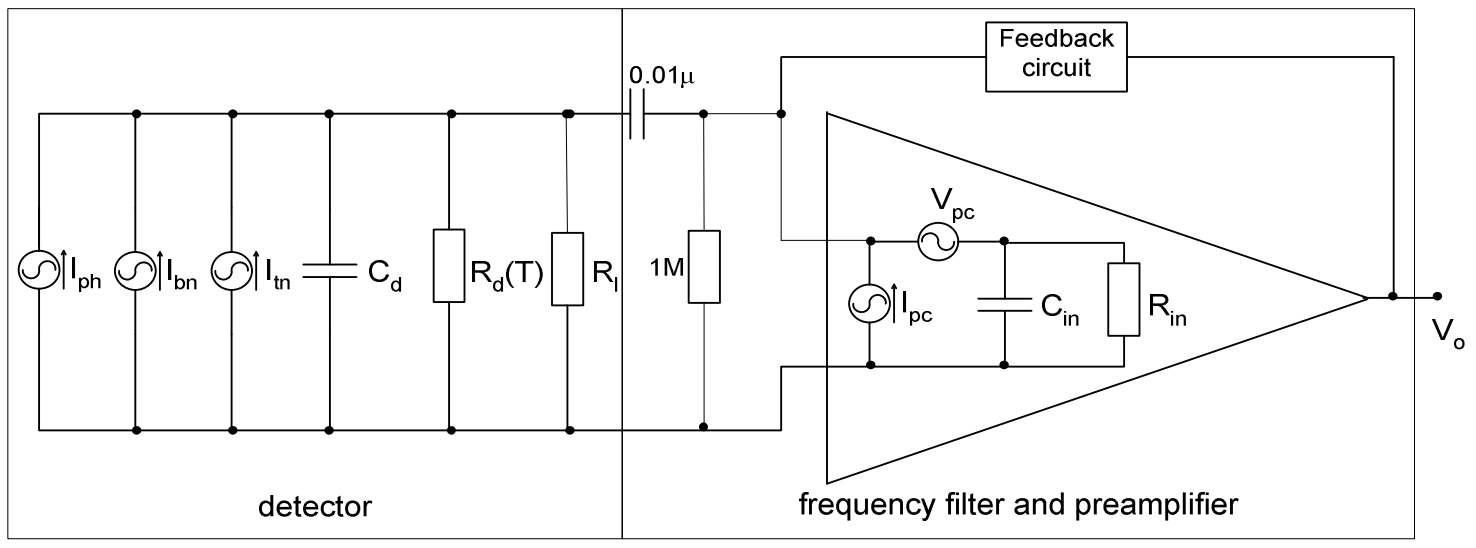

Fig. 3. General noise model of the detector and conditioning circuit 
The case material, the surface ruggedness and the colour, the absolute temperature, the type $(\mathrm{PbS})$ and active area of the applied detector, the chopping frequency of the detector are some of these, for example. Whole metal surfaces of the moisture meter are blacked by the blackening material for its high and near uniform absorption. The blackening material is chosen to provide the incident background radiation to the detector area to be minimal as much as possible. For example, the peak radiated energy is depending on absolute temperature of the environment and varies when it is changing. The background noise can be reduced by narrowing the detector's field of view. Furthermore, the incident background radiation flux which enters vertically to the detector area can be achieved by designing appropriate moisture meter construction. This noise can be weighty in the case of a very low signal and very low detector noises, but due to the discrete nature of radiation, which is composed of photons arriving randomly in time, this noise can be generated by actual desired signal photons. Absorbed photons composed either in the background flux or in the desired signal flux produce photoelectrons at random intervals, and its variation in current appears as noise. When that occurs, the detector system operates in a background limited performance mode (BLIP). The thermal component is included in the background radiation yet but it has a different nature of the noise generation. Photons are absorbed in a way they can produce both positive and negative charge carriers. Some of the free carriers may recombine before they are collected and thermal excitation may generate additional carriers. Both, the generation and recombination occur randomly, resulting in noise fluctuations in the output current and the output voltage of the detector. It induces the thermally excited carriers' generation-recombination in semiconductors so that the thermal generated noise is emerging in the detector. This noise can be ranked in the third type, which is named as detector noises together with the other one which is generated by the same principle due to detector temperature over $0 \mathrm{~K}$. The reduction of the thermal excited carriers as well as the decreasing of the detector noise can be ensured by the cooling detector. The noise and thermal drift characteristics of the detector vary from one end to the other in the same type, which characterise the internal processes that govern the operation of the detector. This is due to the great disparity between the values of the parameters with respect to the typical ones of the model. Using the particular calibration data provided by manufacturer, it is possible to extract the detector noise spectrum, approximately. Because the detector does not provide some internal amplification of the photocurrent, just as photo multipliers have. The lowest measurable signal is determined by the processing circuitry behind the detector. The basic aim of this paper is to find out whether the signal value of detector can bi reduced in this measurement system by the appropriate choice of chopper frequency and corresponding processing circuit design. Noises which turned up by the processing circuits are unattended thereby. It is assumed that the spectral noise level generated by the conditioning system compared with the contribution of the sensor across all the ranges of useful frequencies is negligible.

\section{FREQUENCY CHARACTERISTICS}

When the load resistance $R_{1}$ and dark resistance $R_{d}$, Fig.2, are equivalent, the maximum signal can be obtained. The output signal level versus $R_{l} / R_{d}$ ratio is shown in Fig.5. The frequency responses of the useful signal can be obtained from the following expression

$$
V(f)=\frac{V_{0}}{\sqrt{1+(2 \pi f \tau)^{2}}},
$$

where $V(f)$ and $V_{0}$ are the voltage signal from the detector and DC response respectively. $\mathrm{f}$ is the chopping frequency in $[\mathrm{Hz}]$ and $\tau$ is time constant in $[\mu \mathrm{s}]$. The noises do not change so simple with frequencies as a useful signal. PbS detector as many other photoconductive detectors exhibit two noise components, white noise is the first and flicker is the second one. White noise has uniform spectral power

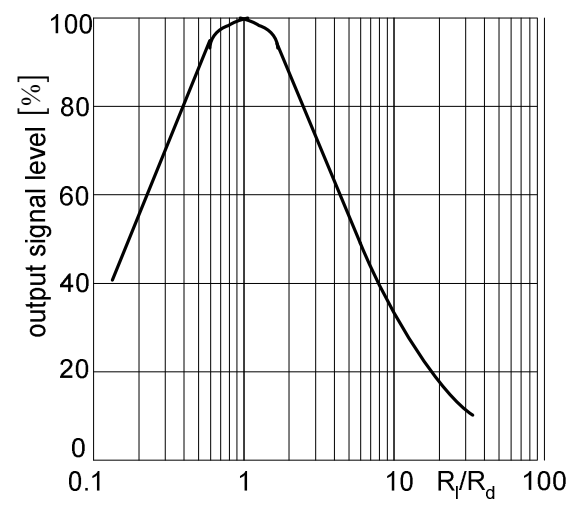

Fig. 5. Output signal level versus $R_{l} / R_{d}$ ratio

density and is not important what detection frequency is used, only the bandwidth. The detector white noise depends on the square root of the noise bandwidth rather than on the bandwidth itself and is usually given in $\mathrm{A} / \sqrt{\mathrm{Hz}}$ or in $\mathrm{V} / \sqrt{\mathrm{Hz}}$. Compared to the white noise, the flicker noise component depends inversely on the frequency $(1 / \mathrm{f})$. This component is dominant at low frequencies and decreases with increasing frequency. At high frequencies, the detector noise actually decreases in the same way as signal decreases. The typical frequency characteristics at 
room temperature of a $\mathrm{PbS}$ detector are shown in Fig. 6.

The detector performance and $\mathrm{S} / \mathrm{N}$ ratio can be

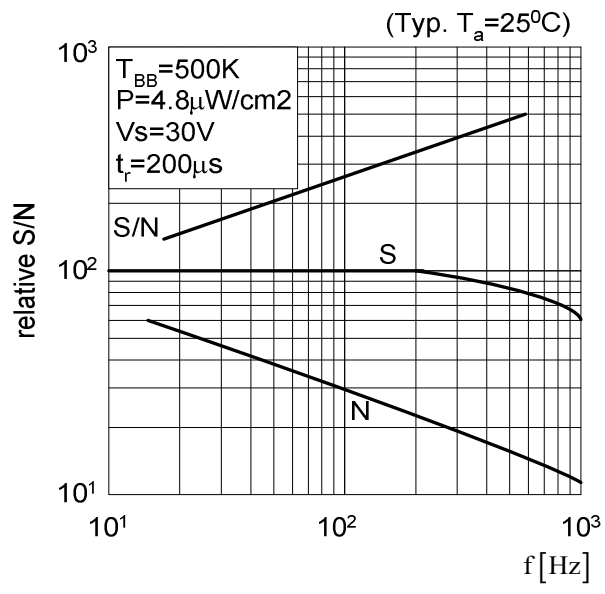

Fig. 6. $\mathrm{S} / \mathrm{N}$ ratio versus chopping frequency maximised by the choice of chopping frequency at

$$
\mathrm{f}=\frac{1}{2 \pi \tau}
$$

The time constant $\tau$ depends on the detector temperature and it increases when the detector is cooled. Since the white noise has a constant value at all frequencies, the performance of the measuring system can be increased by narrowing frequency bandwidth.

\section{CONCLUSION}

The NIR moisture meter measuring system based on the cooled $\mathrm{PbS}$ photoconductive detector is developed by the authors and theoretically analysed considering different detector temperature and chopping frequency. This system is checked by the measuring reflection from corn samples of the different moisture content and with band pass optical filter for wavelength $1940 \mathrm{~nm}$ which is embedded in the rotational wheel. One obtained output signal from the preamplifier is measured at the chopping frequency $175 \mathrm{~Hz}$ and room temperature $25^{\circ} \mathrm{C}$ and shown in Fig. 7. The detector temperature is measured by the embedded thermistor. Determination of the functional dependence of moisture content $[\%]=f$ (Absorbance-reflectance) $[\%]$ which is typical for every substance included into the experimental phase along with the corresponding application of the moisture meter working by using the thermo-gravimetric procedure. Owing to different measured signals the possible feasibility of the maximum $\mathrm{S} / \mathrm{N}$ ratio must be previously investigated. Many problems can be anticipated when the signal of the reflected beam is small and its level fails into the levels of the total detector noises. It can be partly solved by increasing the total detector load and partly by increasing the detectivity via the decreasing of the

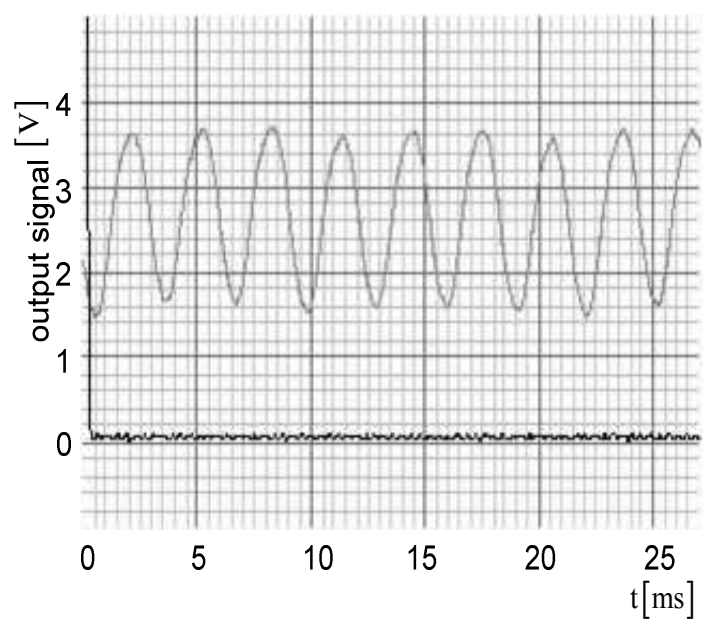

Fig. 7. Measured output signal from preamplifier

temperature with the thermoelectrical cooler which is embedded in the detector. The output signal from detector can be increased by increasing the total density the incident radiation which is captured.

\section{REFERENCES}

[1] V. Corluka, M. Filic, M. Mesic, Z. Valter, "Near infrared based moisture meter", Elmar-2004, pp. 412417, ISBN 1334-2630, Zadar (Croatia), June 2004.

[2] V. Corluka, M. Filic, M. Mesic, Z. Valter, "Optoelectronic moisture measurement", 3rd ATDC 2004, pp. 303-308, ISBN 953-6114-68-2, Split (Croatia), June 2004.

[3] V. Corluka, M. Filic, Z. Valter, "Development of one infrared moisture meter", $15^{\text {th }}$ DAAAM International Symp. 'Intelligent Manufacturing \& Automation', Wien (Austria), pp. 081-082, ISBN 3-901509-42-9, November 2004

[4] M. Mesic, M. Filic, Z. Valter, "Experience with Detectors for Infrared Moisture Measuring", $6^{\text {th }}$ ISEMA 2005 Int. Conf. on 'Electromagnetic Wave Interaction with Water and Moist Substances", Weimar (Germany), pp. 535-540, June 2005

[5] Z. Bielecki, W. Kolosowski, R. Dufrene, M. Borejko, "Low noise optical receivers", $11^{\text {th }}$ GAAS Symposium, Munich (Germany), pp. 137-140, 2003.

[6] W. Leschnik, "Feuchtemessung an BaustoffenZwischen Klassik und Moderne", DGZfPProceedings, Berlin (Germany), pp.1-23, BB 69-h2, Feuchtetag 1999.

[7] H. Günzler, \& H.-U. Gremlich, IR-Spektroskopie, Wiley-VCH Verlag GmbH \& Co. KGaA, ISBN 3527-30801-6, Weinheim, 2003.

[8] P. Geladi and E. Dabbak: "An overview of chemometrics applications in near infrared spectrometry", Journal of Near Infrared Spectroscopy, 3(3), pp. 119-132, 1995.

[9] K.A.Büscher, W.Wild, H.Wiggenhauser, "Feuchtemessung mit infraoptischen Methoden", DGZfP-Proceedings, Berlin (Germany), BB 69-m2, Feuchtetag 1999. 